\title{
INNOVATIVE WAY TO RECOVER LIQUID COSMETICS
}

\author{
S. Wawrzyniak* ${ }^{*}$ K. Peszyński**
}

\begin{abstract}
The paper presents a test stand that enables the visualization of the contact area of two liquids of different viscosities during the flow in the pipeline. The analysis of the test results allows to determine the degree of mixing. The purpose of the conducted research was to determine the possibility and quantity of recovery of the completed product in the pipeline while washing the tank during the technological process, before production of a new product
\end{abstract}

Keywords: viscosity, liquid flow, mixing of liquids.

\section{Introduction}

In the literature, there are many papers on research related to the deliberate mixing of products with different viscosities and densities in order to obtain a product with specific properties, e.g. emulsions and others e.g. (Gallettin Ch. et al., 2015). No work to avoid mixing two liquids in pipes was found.

Produced cosmetics are characterized by various physical parameters. From the point of view of the tests presented below, the most important parameters are viscosity and partly density. In industrial conditions, different cosmetics as shampoos, conditioners, moisturizing emulsions, perfumes, etc., are produced in the same tanks. When changing the production profile, part of the product remains in the pipeline between the picking pump and the final part of the line, i.e. the dispensing system for the product packaging, e.g. for bottles. Previously, the product remaining in the pipeline is in many cases partly recovered using a intermediate mechanical unit, pushed over straight sections of the pipeline, Fig. 1a.

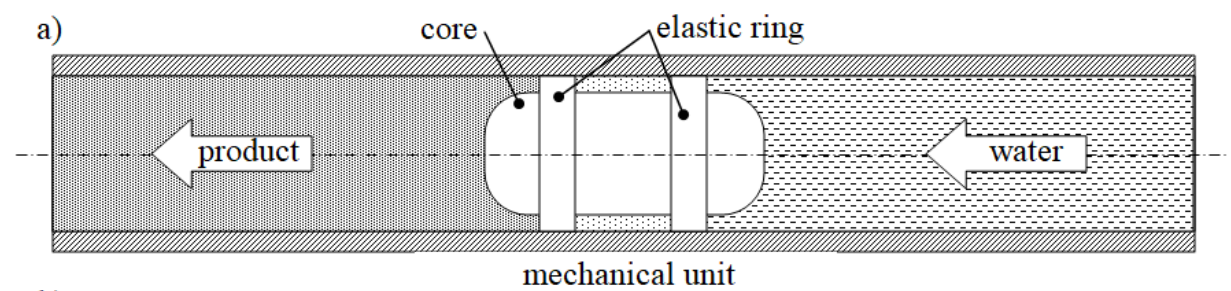

b)

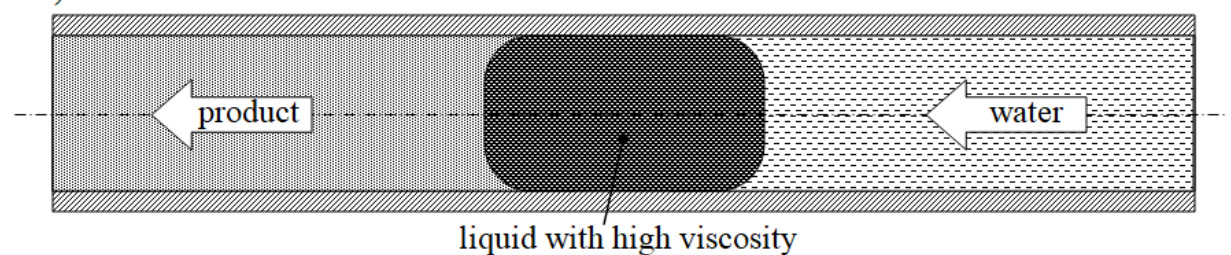

Fig. 1:The idea of recovering liquid cosmetics: a) old, b) innovative.

This unit consists of a metal core and elastic elements sized to the diameter of the pipeline to seal the parts in front of and behind the unit. This design of the unit allows movement inside the pipeline on

Assist. prof. Sylwester Wawrzyniak, PhD., UTP University of Science and Technology, Al. prof. S. Kaliskiego; 85-796 Bydgoszcz; PL, sylwester.wawrzyniak@utp.edu.pl

** Assoc. prof. Kazimierz Peszyński, PhD, UTP University of Science and Technology, Al. prof. S. Kaliskiego; 85-796 Bydgoszcz; PL, kazimerz.peszynski@utp.edu.pl 
straight sections and elbows with a gentle arches. The subject of the research is the possibility of using a liquid intermediate element separating water from the product while cleaning the pipeline. During the preliminary studies presented in this paper, the mixing of water and liquid with a higher viscosity during the flow in the research stand was studied. An intermediary unit of liquid of high viscosity between the two liquids was omitted.

\section{Research stand}

Because it is not possible to carry out tests on a real installation, a model of the pipeline basic part was built. Plexiglas pipes were used - outer diameter $30 \mathrm{~mm}$ and inner diameter $24 \mathrm{~mm}$. Transparent pipes enable observation of the product and water contact area during the flow. Due to the difficulties in making $90^{\circ}$ elbows with the appropriate ratio of diameter to radius arches, standard hydraulic elbows made of polypropylene were used.

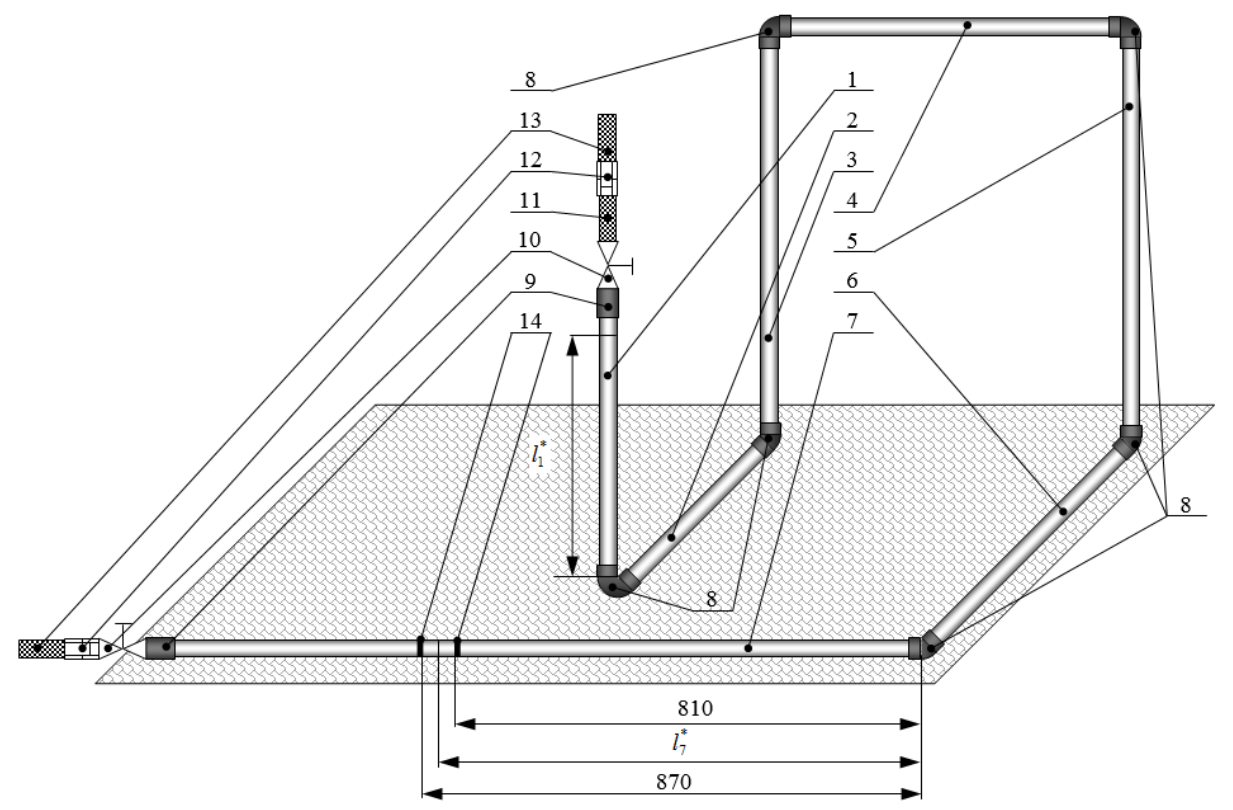

Fig. 2:Diagram of the constructed test stand.

Fig. 2 presents a diagram of the constructed pipeline model used for experimental research, which consists of: $1 \div 7-$ straight pipe with length $l_{1}=0.45 \mathrm{~m}, l_{2}=l_{3}=l_{5}=0.7 \mathrm{~m}, l_{4}=0.6 \mathrm{~m}, l_{6}=1 \mathrm{~m}, l_{4}=1.3 \mathrm{~m}$, 8 - six elbows, 9 - two connectors, 10 - two valves, 11, 13 - rubber hoses, 12 - two connection fittings, 14 - observation window between two tape strips. Filling by the tested liquid of the first section was on the length $l_{1}^{*}=0,43 \mathrm{~m}(1 \mathrm{~cm}$ below the end of the connector $)$, sections $l_{i}(i=2,3, \ldots, 6)$ were completely filled, while the last section on length $l_{7}^{*}=0,84 \mathrm{~m}$. Therefore, the total length of the straight tube sections filled with the test substance was:

$$
l=l_{1}^{*}+l_{2}+l_{3}+l_{4}+l_{5}+l_{6}+l_{7}^{*}=0.43+3 \cdot 0.7+0.6+1.0+0.84=4.97 \mathrm{~m}
$$

The volume of the pipeline (rectilinear pipes) without elbows:

$$
V_{\text {pipe }}=\pi \frac{D_{\text {in }}^{2}}{4} l=\pi \frac{(0.024 \mathrm{~m})^{2}}{4} 4.97=0.00225 \mathrm{~m}^{3}
$$

The principle of calculating the volume of a curved part of the elbow is explained in Fig. 3 a), d), e). The volume of liquid filling the elbow is 0.25 volume of torus. The test installation contains 6 elbows, therefore their total volume for the radius $r=0.0155 \mathrm{~m}$ is

$$
V_{\text {6elb }}=6 V_{\mathrm{elb}}=3 \pi^{2} r^{3}=3 \cdot \pi^{2} \cdot 0.00155^{3}=0.00011 \mathrm{~m}^{3}
$$

Therefore, the total amount of filled substance in test installation is

$$
V_{\mathrm{s} \_ \text {in }}=V_{\text {pipe }}+V_{\text {6elb }}=0.002248+0,00011 \approx 0.00236 \mathrm{~m}^{3}
$$




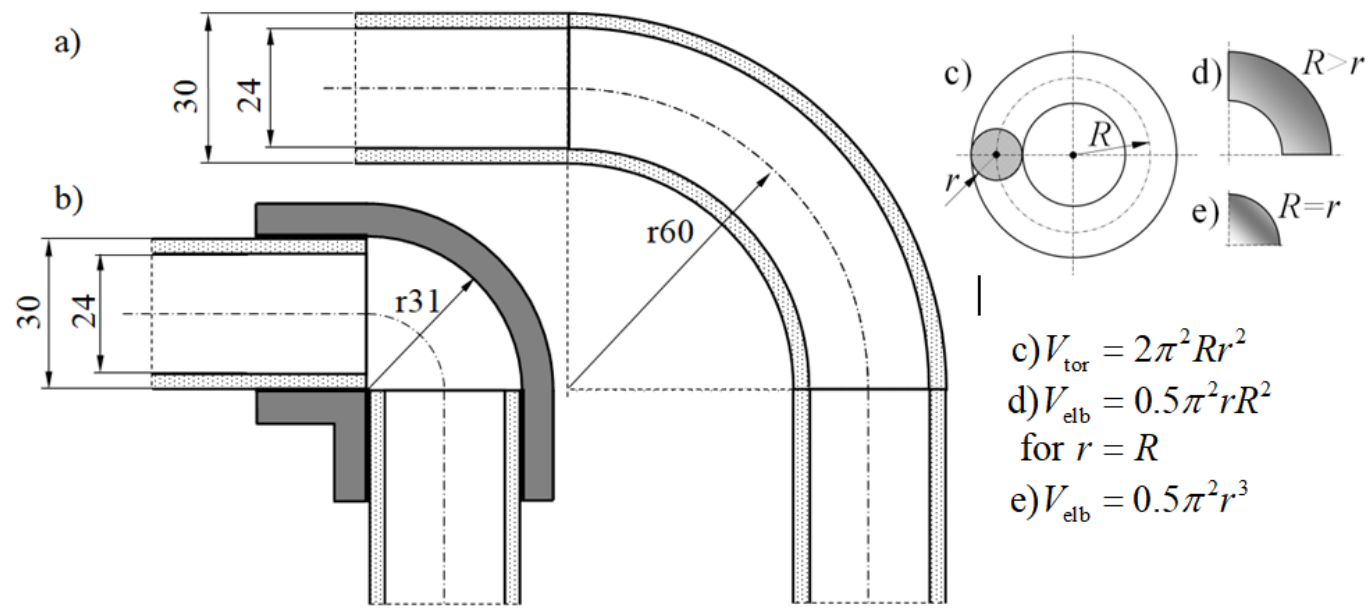

Fig. 3:Elbow: a) real, b) applied, c) torus idea, d) classic elbow e) used elbow.

Flow in the test installation took place in worse conditions than in the actual installation, due to less flowfriendly elbows, see Fig. 4,

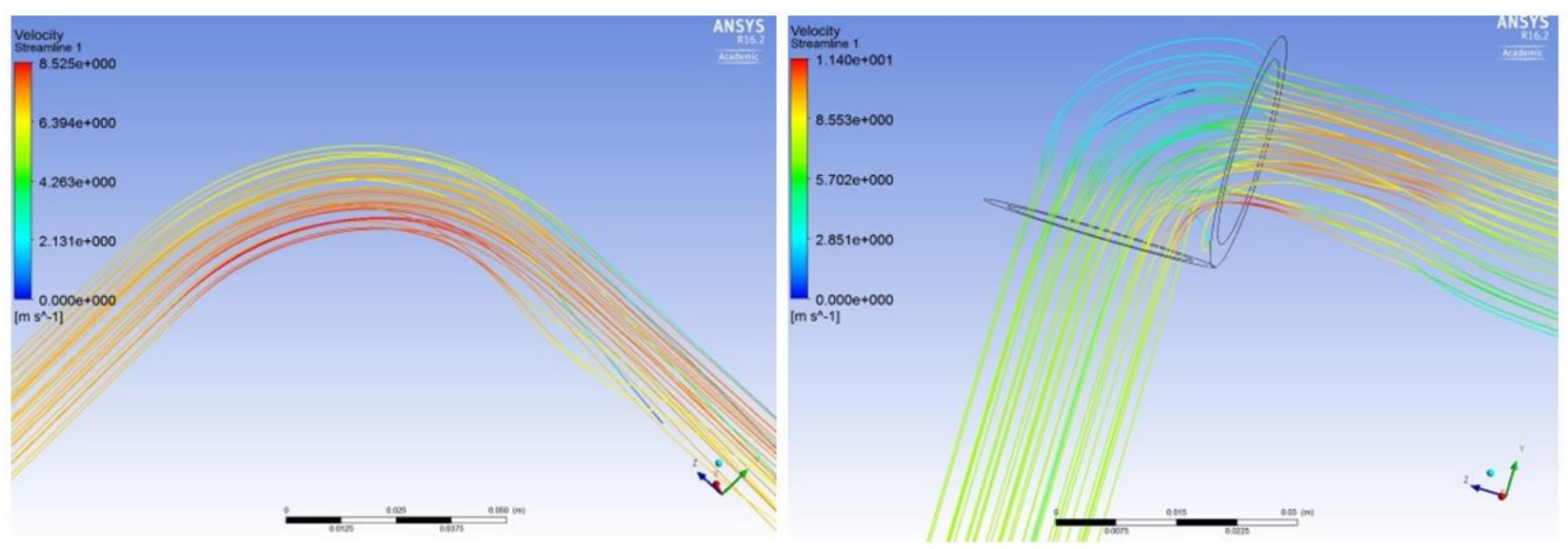

Fig. 4:Elbow: a) actual installation, b) test installation.

Measurements of flow velocity in the tested pipeline were carried out by means of an electromagnetic flowmeter that allows non-invasive measurement of the flow velocity of fluids in pipelines. An ultrasonic portable flow meter from Endress+Hauser, model Proline Prosonic Flow 93T, was used. The measuring range of the flow meter is $0 \ldots 452,389 \mathrm{~m}^{3} / \mathrm{h}$. The measurements were carried out at an ambient temperature of $21^{\circ} \mathrm{C}$. In order to maintain the similarity of the flow to the flow in the actual pipeline, the velocity values in the model pipeline were calculated. The mass flow rate in the real system is $\dot{m}_{\mathrm{a}}=5 \mathrm{~kg} \cdot \mathrm{s}^{-1}$. The internal diameter of the pipeline is $78 \mathrm{~mm}$. Calculation of the flow rate in the actual pipeline (index 'a' means actual):

$$
v_{\mathrm{a}}=\frac{m_{\mathrm{a}}}{\rho A_{\mathrm{a}}}=\frac{5}{1010 \cdot 0.00478}=1.036 \mathrm{~m} \cdot \mathrm{s}^{-1}
$$

While maintaining the same flow velocity in the test pipeline, a mass flow rate output was obtained:

$$
\dot{m}=A_{\mathrm{t}} \cdot \rho \cdot v=0.000452 \cdot 1010 \cdot 1.036=0.4734 \mathrm{~kg} \cdot \mathrm{s}^{-1}
$$

In order to obtain the required flow rate, the flow at the outlet from the pipeline was throttled. Highresolution GOPro sport camera recording images at 25 frames per second was used to record images. The measurements were carried out five times for each viscosity of the tested product. In order to ensure correct visualization of the flow and the area of mixing of the product and water, the water used for pushing the product was dyed blue with a dye, which was potassium permanganate (see Fig. 5). 


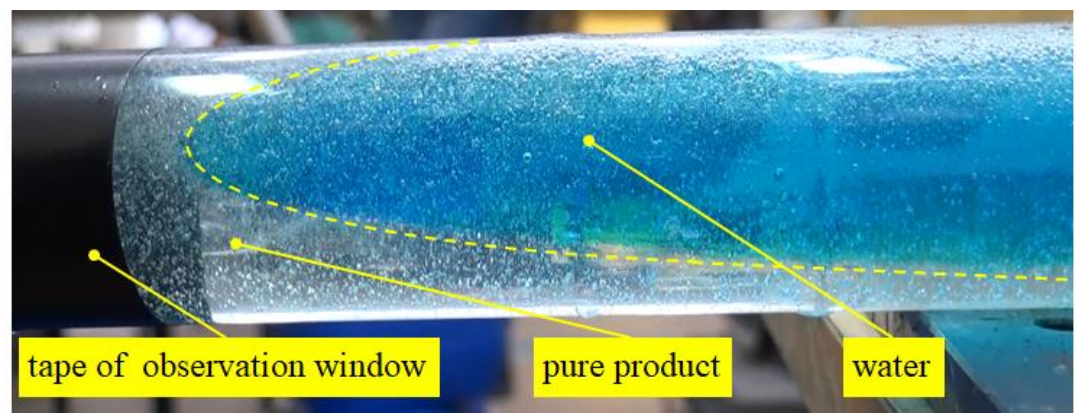

Fig. 5: View of the filled pipeline after stopping the flow at the control point.

The water inflow valve was closed when the presence of stained water was clearly visible at the observation window of the tested pipeline (see Fig. 2, item 14). The product that emerged from the pipeline was weighed to determine the percentage of product recovered.

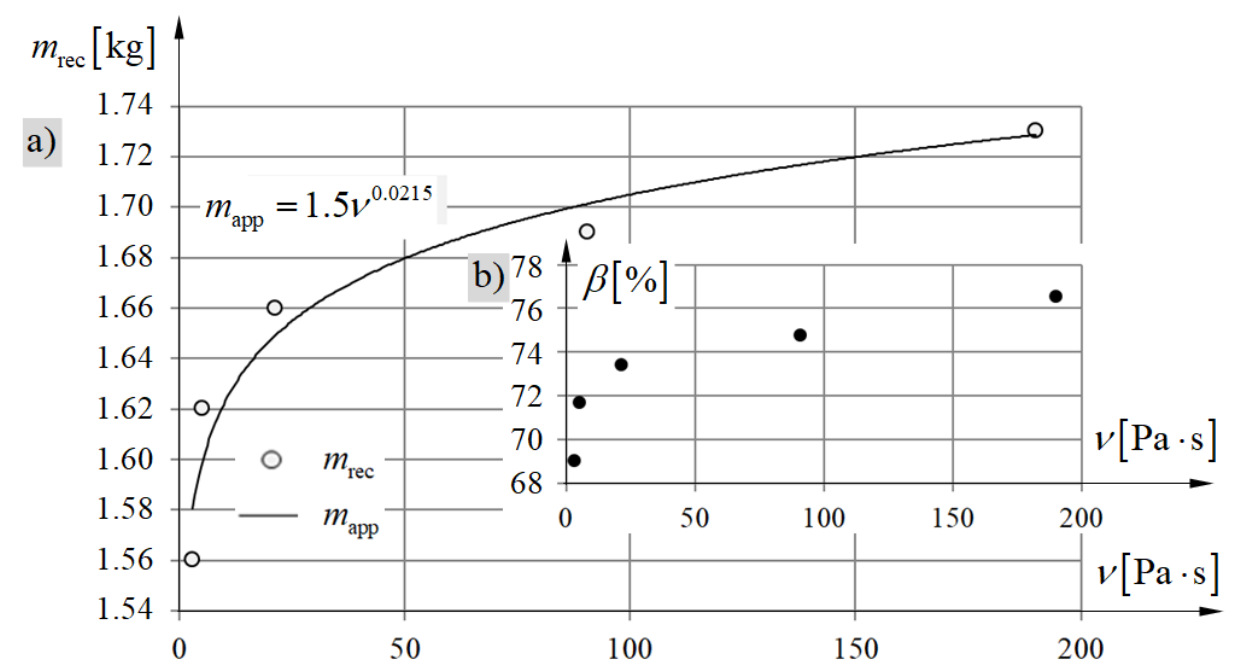

Fig. 6:Recovered product mass: a) absolute value, b) percentage of total mass.

Fig. 6a) shows the test results for a test installation, where $m_{\text {rec }}$ is the mass of the recovered product from the total mass $m_{\text {total }}=2.26 \mathrm{~kg}$ contained in the pipeline. In Fig. $6 \mathrm{~b}$ ) the relative share $\beta$, counted from formula (7), of the recovered product is shown.

$$
\beta=m_{\text {rec }} / m_{\text {total }} \quad \%
$$

An empirical formula, determining the amount of recovered mass produced as a function of viscosity, was also determined:

$$
m_{\text {app }}=1.5 v^{0.00215} \mathrm{~kg}
$$

\section{Conclusions}

Results analysis to conclude that this method can be successfully applied in industrial conditions. When using this method, the viscosity of the product should be taken into consideration, which significantly affects the amount of product recovered. Formula (8) is only valid for a specific technological installation that was a model for a research installation.

\section{Acknowledgement}

Authors received institutional support BS 16/2013 granted by Faculty of Mechanical Engineering of UTP University in Bydgoszcz, Poland.

\section{References}

Gallettin Ch., Arcolini G., Brunazzi E. and Mauri R. Mixing of binary fluids with composition-dependent viscosity in a T-shaped micro-device, Chemical Engineering Science 123 (2015) pp. 300-310. 